\title{
Industry news: 2020 high-impact publications in the BDM area
}

\author{
Liang $\mathrm{Ma}^{1,2}$ (D) \\ Received: 22 December 2020 / Accepted: 22 December 2020 / Published online: 5 January 2021 \\ (c) Zhejiang University Press 2021
}

\begin{abstract}
Although the world in 2020 is suffering from the COVID-19 pandemic, developments in bio-design and manufacturing (BDM) have not ceased. We have selected the most exciting progress in this flourishing area throughout 2020 from the three-dimensional (3D) bioprinting of different functional micro-organoids including kidneys to the manufacture of 3D tissues in the infinite universe. Remarkable technical breakthroughs have been seen in this area including noninvasive in vivo 3D bioprinting, intravital 3D bioprinting, single-cell printing, and rapid prototyping. Significant biomedical applications including human blood-brain barrier platforms, the noninvasive treatment of type 2 diabetes (T2D), and corpus cavernosa reconstructions have further benefited medical care development and our overall quality of life.
\end{abstract}

\section{Nature Communications: Microengineered human blood-brain barrier platform for understanding nanoparticle transport mechanisms}

An article titled 'Microengineered human blood-brain barrier platform for understanding nanoparticle transport mechanisms [1]' published by YongTae Kim's research team from the Georgia Institute of Technology (USA) in Nature Communications provides us with a microphysiological platform that recapitulates the key structure and function of the human BBB and enables 3D mapping of nanoparticle distributions in vascular and perivascular regions. This model captures precisely 3D nanoparticle distributions at the cellular level and demonstrates distinct cellular uptake and blood-brain barrier (BBB) penetrations through receptor-mediated transcytosis.

Liang Ma

liangma@zju.edu.cn

1 State Key Laboratory of Fluid Power and Mechatronic Systems, Zhejiang University, Hangzhou 310058, People's Republic of China

2 School of Mechanical Engineering, Zhejiang University, Hangzhou 310058, People's Republic of China
This BBB platform also presents a complementary in vitro model for animal approaches for prescreening drug candidates for the treatment of neurological diseases.

\section{Cell Metabolism: Exposure to static magnetic and electric fields treats type 2 diabetes}

An article entitled 'Exposure to Static Magnetic and Electric Fields Treats Type 2 Diabetes [2]' was published by Carter and Huang et al. from the University of Iowa in Cell Metabolism. This study demonstrates a remarkable redox-modulating phenomenon that exploits endogenous electromagneticreceptive mechanisms for the noninvasive treatment of T2D as well as potentially other redox-related diseases.

\section{Nature Materials: Cellular extrusion bioprinting improves kidney organoid reproducibility and conformation}

Melissa H. Little's research team from Australia published an article entitled 'Cellular extrusion bioprinting improves kidney organoid reproducibility and conformation [3]' in Nature Materials. In the study, the authors apply extrusion-based 3D cellular bioprinting to deliver rapid and high-throughput generation of kidney organoids with highly reproducible cell number and viability. Automated extrusion-based bioprinting for kidney organoid production delivers improvements in throughput, quality control, scale and structure, facilitating in vitro and in vivo applications of stem cell-derived human kidney tissue.

\section{Nature Materials: Recapitulating macro-scale tissue self-organization through organoid bioprinting}

An article titled 'Recapitulating macro-scale tissue selforganization through organoid bioprinting [4]' was published by Matthias P. Lutolf's team from the Ecole Polytechnique Fédérale de Lausanne. This study introduces a 3D bioprinting 
concept that uses organoid-forming stem cells as building blocks that can be deposited directly into extracellular matrices conducive to spontaneous self-organization. This study shows how biofabrication and organoid technology can be merged to control tissue self-organization from millimeter-to-centimeter scales, opening new avenues for drug discovery, diagnostics, and regenerative medicine.

\section{Science Advances: Magnetic levitational bioassembly of 3D tissue construct in space}

Vladimir A. Mironov from the Laboratory for Biotechnological Research '3D Bioprinting Solutions' and other researchers published an article entitled 'Magnetic levitational bioassembly of 3D tissue construct in space [5]' in Science Advances. In this research, 3D tissue constructs were biofabricated for the first time in space under microgravity from tissue spheroids consisting of human chondrocytes. These data strongly suggest that scaffold-free formative biofabrication using magnetic fields is a feasible alternative to traditional scaffold-based approaches, hinting at a new avenue of research that could significantly advance tissue engineering. Magnetic levitational bioassembly in space can also advance space life science and space regenerative medicine.

\section{Science Advances: Noninvasive in vivo 3D bioprinting}

An article titled 'Noninvasive in vivo 3D bioprinting [6]' published by Yuwen Chen et al. from Sichuan University in Science Advances demonstrates digital near-infrared (NIR) photopolymerization (DNP)-based 3D printing technology that enables the noninvasive in vivo 3D bioprinting of tissue constructs. Thus, without surgery implantation, a personalized ear-like tissue constructs with chondrification and a muscle tissue repairable cell-laden conformal scaffold were obtained in vivo. This work provides a proof of concept of noninvasive in vivo $3 \mathrm{D}$ bioprinting.

\section{Nature Biomedical Engineering: Intravital three-dimensional bioprinting}

Nicola Elvassore's team from the University of Padova published an article titled 'Intravital three-dimensional bioprinting [7]' in Nature Biomedical Engineering. The study shows that 3D cell-laden photosensitive polymer hydrogels can be bioprinted across, and within, tissues of live mice, using bio-orthogonal two-photon cycloaddition and crosslinking of polymers at wavelengths longer than $850 \mathrm{~nm}$. Similarly, $3 \mathrm{D}$ bioprinting of donor-muscle-derived stem cells under the epimysium of hindlimb muscle in mice leads to the de novo formation of myofibres in the mice. Intravital 3D bioprinting might serve as an in vivo alternative to conventional bioprinting.

\section{Advanced Materials: High-definition single-cell printing: Cell-by-cell fabrication of biological structures}

In an article entitled 'High-definition single-cell printing: Cell-by-cell fabrication of biological structures [8]' published by Pengfei Zhang and Adam R. Abate from the University of California in Advanced Materials, a novel microfluidic technology called high-definition single-cell printing was presented that can accurately print single cells from a mixture of multiple candidates. This bioprinter employs a highly miniaturized microfluidic sorter to deterministically select single cells of interest for printing, achieving an accuracy of $\approx 10 \mu \mathrm{m}$ and speed of $\approx 100 \mathrm{~Hz}$. This approach was shown to work well by fabricating intricate cell patterns with pre-defined features through selective single-cell printing. This printer was then used to synthesize well-defined spheroids with controlled composition and morphology. The speed, accuracy, and flexibility of this approach to advance bioprinting will enable new studies in organoid science, tissue engineering, and spatially targeted cell therapies.

\section{Nature Communications: Functional reconstruction of injured corpus cavernosa using 3D-printed hydrogel scaffolds seeded with HIF-1a-expressing stem cells}

Research led by Xuetao Shi from the South China University of Technology and Chuanbin Mao from the University of Oklahoma published an article titled 'Functional reconstruction of injured corpus cavernosa using 3D-printed hydrogel scaffolds seeded with HIF-1 $\alpha$-expressing stem cells [9]' in Nature Communications. These authors constructed heparincoated 3D-printed hydrogel scaffolds seeded with hypoxia inducible factor-1 alpha (HIF-1 alpha)-mutated musclederived stem cells (MDSCs) to develop bioengineered vascularized corpora. These bioengineered corpora scaffolds repair defects and recover penile erectile and ejaculation function successfully. This functional recovery restores the reproductive capability of injured male rabbits and demonstrates that the 3D-printed hydrogels with angiogenic cells hold great promise for penile reconstruction to restore male reproductive capability. 


\section{Nature Biomedical Engineering: The rapid prototyping of soft bioelectronic implants for use as neuromuscular interfaces}

Ivan R. Minev from the Technische Universität Dresden as well as Pavel Musienko from Saint-Petersburg State University and other researchers published an article titled 'Rapid prototyping of soft bioelectronic implants for use as neuromuscular interfaces [10]' in Nature Biomedical Engineering. The study shows that soft biocompatible materials can be rapidly printed for the on-demand prototyping of customized electrode arrays well-adjusted to specific anatomical environments, functions, and experimental models. These printed bioelectronic interfaces allow for long-term integration and functional stability. This technology might enable personalized bioelectronics for neuroprosthetic applications.

\section{Bio-Design and Manufacturing published a special issue-'In vitro tissue models'}

Yu Shrike Zhang from Harvard Medical School and Ali Khademhosseini from Terasaki Institute for Biomedical Innovation (TIBI) organized the special issue focus on the in vitro tissue models for BDM in 2020. From the Editorial titled 'Engineering in vitro Human Tissue Models through Bio-Design and Manufacturing [11]' and a set of articles in the issue, we can know that the in vitro tissue models manufactured by 3D bioprinting or microfluidic based organ-on-chips technologies can provide a powerful tool for personalized medicine.

\section{Compliance with ethical standards}

Conflict of interest The author declares that he has no conflict of interest.

Ethical approval This article does not contain any studies with human or animal subjects performed by the author.

\section{References}

1. Ahn SI, Sei YJ, Park HJ, Kim J, Ryu Y, Choi JJ, Sung HJ, Macdonald TJ, Levey AI, Kim Y (2020) Microengineered human blood-brain barrier platform for understanding nanoparticle transport mechanisms. Nat Commun 11:175. https://doi.org/10.1038/ s41467-019-13896-7
2. Carter CS, Huang SC, Searby CC, Cassaidy B, Miller MJ, Grzesik WJ, Piorczynski TB, Pak TK, Walsh SA, Acevedo M, Zhang Q, Mapuskar KA, Milne GL, Hinton AO Jr, Guo DF, Weiss R, Bradberry K, Taylor EB, Rauckhorst AJ, Dick DW, Akurathi V, Falls-Hubert KC, Wagner BA, Carter WA, Wang K, Norris AW, Rahmouni K, Buettner GR, Hansen JM, Spitz DR, Abel ED, Sheffield VC (2020) Exposure to Static Magnetic and Electric Fields Treats Type 2 Diabetes. Cell Metab 32:1076. https://doi. org/10.1016/j.cmet.2020.09.012

3. Lawlor KT, Vanslambrouck JM, Higgins JW, Chambon A, Bishard K, Arndt D, Er PX, Wilson SB, Howden SE, Tan KS, Li F, Hale LJ, Shepherd B, Pentoney S, Presnell SC, Chen AE, Little MH (2020) Cellular extrusion bioprinting improves kidney organoid reproducibility and conformation. Nat Mater. https://doi. org/10.1038/s41563-020-00853-9

4. Brassard JA, Nikolaev M, Hubscher T, Hofer M, Lutolf MP (2021) Recapitulating macro-scale tissue self-organization through organoid bioprinting. Nat Mater 20:22-29. https://doi.org/10.1038/ s41563-020-00803-5

5. Parfenov VA, Khesuani YD, Petrov SV, Karalkin PA, Koudan EV, Nezhurina EK, Pereira FD, Krokhmal AA, Gryadunova AA, Bulanova EA, Vakhrushev IV, Babichenko Ii, Kasyanov V, Petrov OF, Vasiliev MM, Brakke K, Belousov SI, Grigoriev TE, Osidak EO, Rossiyskaya EI, Buravkova LB, Kononenko OD, Demirci U, Mironov VA (2020) Magnetic levitational bioassembly of 3D tissue construct in space. Sci Adv 6:eaba4174. https://doi. org/10.1126/sciadv.aba4174

6. Chen Y, Zhang J, Liu X, Wang S, Tao J, Huang Y, Wu W, Li Y, Zhou K, Wei X, Chen S, Li X, Xu X, Cardon L, Qian Z, Gou M (2020) Noninvasive in vivo 3D bioprinting. Sci Adv 6:eaba7406. https://doi.org/10.1126/sciadv.aba7406

7. Urciuolo A, Poli I, Brandolino L, Raffa P, Scattolini V, Laterza C, Giobbe GG, Zambaiti E, Selmin G, Magnussen M, Brigo L, De Coppi P, Salmaso S, Giomo M, Elvassore N (2020) Intravital three-dimensional bioprinting. Nat Biomed Eng 4:901-915. https ://doi.org/10.1038/s41551-020-0568-z

8. Zhang P, Abate AR (2020) High-definition single-cell printing: cell-by-cell fabrication of biological structures. Adv Mater, $\mathrm{p}$ e2005346. https://doi.org/10.1002/adma.202005346

9. An G, Guo F, Liu X, Wang Z, Zhu Y, Fan Y, Xuan C, Li Y, Wu H, Shi X, Mao C (2020) Functional reconstruction of injured corpus cavernosa using 3D-printed hydrogel scaffolds seeded with HIF1alpha-expressing stem cells. Nat Commun 11:2687. https://doi. org/10.1038/s41467-020-16192-x

10. Afanasenkau D, Kalinina D, Lyakhovetskii V, Tondera C, Gorsky O, Moosavi S, Pavlova N, Merkulyeva N, Kalueff AV, Minev IR, Musienko P (2020) Rapid prototyping of soft bioelectronic implants for use as neuromuscular interfaces. Nat Biomed Eng 4:1010-1022. https://doi.org/10.1038/s41551-02000615-7

11. Zhang YS, Khademhosseini A (2020) Engineering in vitro human tissue models through bio-design and manufacturing. Bio-Des Manuf 3:155-159. https://doi.org/10.1007/s42242-02000080-w 